\title{
Fecal microbiota transplantation in children: a brief review
}

\author{
Suchitra K. Hourigan ${ }^{1,2,3,4}$ and Maria Oliva-Hemker ${ }^{2}$
}

There has been a growing interest in fecal microbiota transplantation (FMT) over recent years, in part due to the increasing prevalence of Clostridium difficile infection (CDI) and expanding association of intestinal dysbiosis with a wide range of human diseases. Many adult studies have shown that FMT is an effective treatment for recurrent CDI and may possibly have applications in other illnesses such as inflammatory bowel disease (IBD); however, there is a paucity of data available in children who may differ from adults for many reasons including having a dynamic developing microbiome compared to adults who have a relatively stable microbiome. Here, we review published studies looking at FMT in children, for CDI and IBD, and discuss special considerations needed when conducting FMT in children.

$\mathbf{T}$ he human intestinal microbiome contains at least $10^{14} \mathrm{bac}-$ teria, with estimates of 1,000 to 1,200 bacterial species that exist in symbiosis with their host $(1,2)$. This complex community of microorganisms has vital functions for their host including synthesis of vitamins, fermentation of dietary carbohydrates, metabolism of bile and host hormones, competitive exclusion of pathogens, and the development and maturation of the immune system (3).

Human microbiome research is a rapidly developing field and our knowledge of the association of dysbiosis, or perturbations of the intestinal microbiome, with a wide range of diseases is increasing. These include gastrointestinal conditions such as inflammatory bowel disease (IBD) and irritable bowel syndrome to metabolic conditions such as obesity (4-7). Clostridium difficile infection (CDI) is one of the best characterized illnesses where a decrease in intestinal microbiome diversity allows an overgrowth of $C$. difficile with an increased risk of recurrent infection (8). Based on this, fecal microbiota transplantation (FMT) or the transfer of stool with a presumed healthy diverse microbiome to a patient, has been used to restore microbiome diversity and successfully treat recurrent CDI (9).

Given the increasing prevalence, recurrence, and resistance to standard treatment of CDI $(10,11)$ there has been growing interest in FMT for CDI especially after two randomized controlled trials in adults have shown efficacy $(12,13)$. Given the success of FMT in this one area, and given the appeal and curiosity exhibited by providers and the lay public, it is not surprising, that consideration of FMT for other conditions associated with disturbances of the microbiome is under active discussion (14).

There are known differences in the microbiome of a child, especially in the very young, compared with adults, with initial colonization of the infant gut and dynamic microbiome development over the next few years setting the stage for a relatively stable microbiome in the healthy adult $(15,16)$. However, there is a paucity of data on FMT in children and the associated microbiome changes. Here, we review published studies looking at FMT in children for CDI and IBD and discuss special considerations needed when applying FMT in children given the potential implications of manipulating the developing microbiome.

\section{FMT FOR RECURRENT CDI IN CHILDREN}

The prevalence of CDI in children is increasing (17), with the highest CDI incidence among children 1 to $4 \mathrm{y}$ of age (18). Recurrence of CDI can occur in $20-30 \%$ after initial infection, with the frequency of recurrence rising further after subsequent infections (19-21). Risk factors for recurrent CDI in children include IBD $(22,23)$, malignancy, recent surgery, and the number of antibiotic exposures by class (19). FMT has been shown to be more effective than antibiotics for recurrent CDI in adults $(12,13)$; van Nood et al. (12) showed a cure rate of $81 \%$ after FMT compared with $31 \%$ with vancomycin alone and Cammarota et al. (13) had similar results with resolution of CDI in $90 \%$ of those treated with FMT compared with $26 \%$ treated with vancomycin. However there have been no randomized controlled trials (RCT) in children. In general, there is also paucity of large case series in children to date (24).

In small pediatric case series of up to 10 patients, pediatric case reports and series with both adults and children (25-33), the effectiveness of FMT was high, with the larger pediatric case series reporting a $90-100 \%(25-27,29)$ success rate in treating recurrent CDI (Table 1). Success in pediatric patients has been reported with delivery of FMT to the upper gastrointestinal tract using nasogastric or transpyloric tubes or to

\footnotetext{
'Department of Pediatrics, Inova Children's Hospital, Fairfax, Virginia; ${ }^{2}$ Department of Pediatrics, Johns Hopkins University School of Medicine, Baltimore, Maryland; ${ }^{3}$ Department of Pediatrics, Pediatric Specialists of Virginia, Fairfax, Virginia; ${ }^{4}$ Inova Translational Medicine Institute, Inova Health System, Fairfax, Virginia.

Correspondence: Suchitra K. Hourigan (suchihourigan@gmail.com)

Received 25 September 2015; accepted 23 December 2015; advance online publication 6 April 2016. doi:10.1038/pr.2016.48
} 
Table 1. Pediatric studies of FMT for recurrent Clostridium difficile infection

\begin{tabular}{|c|c|c|c|c|c|c|}
\hline Author & $\begin{array}{c}\text { Year of } \\
\text { publication }\end{array}$ & $\begin{array}{c}\text { Age } \\
\text { (years) }\end{array}$ & Number of children & Route FMT & Cure rate & Complications \\
\hline Kronman et al. (25) & 2015 & $1-13$ & 10 & Nasogastric & $90 \%$ & $\begin{array}{l}\text { Transient vomiting } \\
\text { and mucoid stool }\end{array}$ \\
\hline Russell et al. (26) & 2014 & $1-19$ & 10 & $\begin{array}{l}\text { Nasogastric (2); } \\
\text { Colonoscopy (8) }\end{array}$ & $90 \%$ & None \\
\hline Pierog et al. (27) & 2014 & $1-21$ & 6 & Colonoscopy & $100 \%$ & None \\
\hline Hourigan et al. (29) & 2015 & $6-17$ & 8 & Colonoscopy & $100 \%$ & None \\
\hline Kahn et al. (30) & 2012 & 1 & 1 & Colonoscopy & $100 \%$ & None \\
\hline Wang et al. (31) & 2015 & 1 & 1 & Nasojejunal & $100 \%$ & None \\
\hline Rubin et al. (32) & 2013 & $6-8$ & 2 children with adult series & Upper tract (route unclear) & $50 \%$ & None \\
\hline Kelly et al. (33) & 2014 & $6-16$ & $\begin{array}{c}5 \text { immuno-comprised children } \\
\text { with adult series }\end{array}$ & Not specified for children & $89 \%$ (whole series) & $\begin{array}{l}\text { No infectious } \\
\text { complications }\end{array}$ \\
\hline
\end{tabular}

FMT, fecal microbiota transplantation.

the lower gastrointestinal tract via colonoscopy (25-27). FMT has been reported as unsuccessful in three pediatric patients: a 4-y-old immunosuppressed female with Wilm's tumor who received FMT via NJ tube (25), a 5-y-old boy with underlying IBD who received FMT via colonoscopy (26), and a child between $6-8$ y of age without underlying IBD, and with FMT instillation via the upper tract (32).

Very young children represent a unique population given their dynamic microbiome and physical size. The youngest reported child receiving FMT for CDI is a 13-mo-old infant (31), with a few other reports of cases under the age of $3 \mathrm{y}$ $(25,26,28,30)$. These cases were successful, and FMT for CDI has also been associated with improved growth in young children (28). In this population, one has to be careful about the choice of patient for FMT given the high rate of asymptomatic carriage of $C$. difficile in very young children $(34,35)$; specifically those considered for FMT should have clear symptoms of CDI that resolve or improve with $C$. difficile specific treatment but then recur and also have other causes of diarrhea screened for and ruled out.

Children with underlying IBD have successfully received FMT for recurrent CDI $(25-27,29)$ without reported worsening of their IBD symptoms. Many of these IBD patients, and other reported children were immunosuppressed at the time of FMT $(25,29,33)$. Of note, while children with IBD do have an increased risk of recurrent CDI infection, patients with IBD who are $C$. difficile positive must be selected carefully for FMT, as there is a high rate of $C$. difficile carriage in this population and so symptoms may be due to IBD as oppose to CDI (36).

There have been a small number of reports in adults of complications from FMT for recurrent CDI including worsening colitis in a patient with IBD (37) and bacteremia (38). While mild transient symptoms including abdominal pain, vomiting, and loose stool have been described in pediatric cases, no long-term complications have been reported in children as a result of FMT for CDI; follow up in pediatric case series has been variable, although some patients have been followed for up to 2 y $(25,28)$.
In adults, prior to FMT for recurrent CDI, there is decreased bacterial diversity compared to healthy donors (39-41). After FMT, microbiota diversity increases in fecal samples and microbiome composition shifts towards the healthy donor (39). The intestinal microbiome of children, especially the very young, is known to differ from adults yet there is very little data characterizing associated microbiome changes with FMT in children. Microbiome changes have been reported in a 20mo-old boy (28), with decreased diversity of the microbiome prior to FMT, with a relative abundance of Proteobacteria, with increased diversity after FMT with increasing Bacteroidetes; these are similar changes to those previously described in adults $(39,42)$. Additionally, in a case series of older children increased microbiome diversity after FMT for CDI appears to be sustained in those without IBD, however at 6 mo decreases to pre-FMT baseline in those with IBD, suggesting IBD hostrelated mechanisms may modify fecal microbiome diversity (29).

\section{FMT FOR IBD IN CHILDREN}

IBD has been associated with underlying intestinal dysbiosis, with a decreased diversity of species in Crohn's disease and ulcerative colits (UC) $(4,5)$. In adults, a systematic review of published studies showed that in general FMT in IBD was safe, but efficacy was variable with conflicting results from RCTs (43). For example, one RCT showed superiority of FMT compared with placebo via enema in active UC in inducing remission, defined as a Mayo score $<2$ and endoscopic Mayo score of 0 at week 7 (44). Conversely, a further RCT found no difference in clinical and endoscopic remission for UC following FMT using healthy donors versus autologous fecal transplant (45).

There is limited data for the treatment of IBD in children with FMT (Table 2), with no RCTs to date. Kunde et al. (46) delivered FMT to children and young adults with UC via serial enemas and reported that six of nine patients maintained a clinical response at $1 \mathrm{mo}$, as manifested by a decrease in Pediatric Ulcerative Colitis Activity Index (PUCAI) score of 
Review | Hourigan and Oliva-Hemker

Table 2. Pediatric studies of FMT for inflammatory bowel disease

\begin{tabular}{|c|c|c|c|c|c|}
\hline Author & $\begin{array}{c}\text { Year of } \\
\text { publication }\end{array}$ & $\begin{array}{l}\text { Age } \\
\text { (years) }\end{array}$ & Number of patients & Route FMT & Outcome \\
\hline \multirow[t]{2}{*}{ Kunde et al. (45) } & \multirow[t]{2}{*}{2013} & \multirow[t]{2}{*}{$7-21$} & \multirow[t]{2}{*}{ Ten with ulcerative colitis } & \multirow[t]{2}{*}{ Serial enemas } & -1 could not hold enema \\
\hline & & & & & $-6 / 9$ maintained response at $1 \mathrm{mo}$ \\
\hline Suskind et al. (46) & 2014 & $13-16$ & Four with ulcerative colitis & Single FMT via nasogastric tube & No clinical or laboratory benefit \\
\hline \multirow[t]{2}{*}{ Kellermayer et al. (48) } & \multirow[t]{2}{*}{2015} & \multirow[t]{2}{*}{$14-16$} & \multirow[t]{2}{*}{ Three with ulcerative colitis } & \multirow[t]{2}{*}{ Colonoscopy and serial enemas } & $\begin{array}{l}-3 / 3 \text { endoscopic and histologic } \\
\text { remission at } 2 \mathrm{wk} \text {. }\end{array}$ \\
\hline & & & & & $\begin{array}{l}-3 / 3 \text { symptom free at } 4 \text { wk with } \\
\text { withdrawal immunotherapy }\end{array}$ \\
\hline
\end{tabular}

FMT, fecal microbiota transplantation.

$>15$; the treatment was found to be safe and tolerable although transient mild-moderate side effects were experienced. Conversely, Suskind et al. (47) reported on four children who received a single FMT via NG tube for UC; this was well tolerated but did not show any clinical or laboratory improvement in patients. Suskind et al. in a separate report showed that seven of nine patients receiving FMT via NG tube for the treatment of Crohn's disease showed evidence of engraftment of donor stool microbiome and a clinical response with improvement of Pediatric Crohn's Disease Activity Index score (48). These patients tolerated the procedure without adverse events. Kellermayer et al. (49) reported transient withdrawal of immunotherapy in three children with UC given high frequency serial FMT by colonoscopy and subsequent enemas. Moreover, serial FMT induced a transient engraftment of the donor microbiome in the recipient. Lastly, serial FMT was shown to be effective in treating a 1-y old with early onset colitis, although this patient did experience systemic adverse reactions that were transient (50).

Although FMT trials in IBD, especially in children, are still in their early stages, on the surface the procedure appears to be safe and tolerable although moderate but transient side effects have been reported. The efficacy from reports is variable, but this treatment may offer some benefit in certain patients potentially correlating with a transient improvement in underlying dysbiosis. There are many unanswered questions, both in children and adults, such as the optimal frequency and length of FMT needed, and whether different protocols or routes of administration in those with Crohn's disease and ulcerative colitis or based on disease location are warranted. Moreover IBD is a complex disease with varying phenotypes and genotypes being associated with certain shifts in microbial composition (51); this questions whether patients with differing IBD genotypes and phenotypes would differentially respond to FMT and warrants further investigation.

\section{FMT FOR OTHER CONDITIONS IN CHILDREN}

FMT is being considered for many diseases associated with microbiome perturbations with trials currently being conducted in adults for conditions such as irritable bowel syndrome, metabolic syndrome, and obesity. However, in children, the only published evidence to date for FMT has been in CDI and IBD.

\section{WHEN TO REFER CHILDREN FOR FMT}

Currently, the only indication for which FMT can be used without an investigational new drug application from the Food and Drug Administration is CDI. Guidelines often followed for when children with CDI may need FMT are: (i) In recurring or relapsing CDI, with at least three episodes of mildmoderate infection and failing a 6-8-wk vancomycin taper or at least two episodes of severe CDI resulting in hospitalization and associated with significant morbidity; (ii) In moderate CDI not responding to standard therapy (vancomycin) for at least a week; and (iii) In severe CDI with no response to standard therapy after $48 \mathrm{~h}$ (52). Children with CDI who may need FMT most often fit into the first category of mild-moderate recurring or relapsing CDI. We suggest referring children to a pediatric gastroenterologist or infectious disease specialist by their second recurrence or relapse of infection in order for treatment to be optimized, to try and prevent the need for FMT and to expedite referrals to specialist centers should FMT be required.

\section{SPECIAL CONSIDERATIONS, PRECAUTIONS, AND FUTURE DIRECTIONS FOR FMT IN CHILDREN}

While small studies of FMT in children hold great promise for the treatment of recurrent CDI, and possibly IBD, questions and concerns are raised specifically pertaining to FMT in children. To date, most studies of FMT in children have used an adult stool donor. Children, especially the very young, have a rapidly developing gut microbiome (53) which correlates with development of the immune system and other physiological functions, reaches a stage of stabilization in the first few years of childhood (16) and differs from the microbiome of the elderly (54). As shown in animal models, FMT may have the potential to transfer a pathogenic disease state from donor to recipient (55), which may be more significant in the developing 
microbiome (56). Moreover, in adults, the development of autoimmune disease and rapid weight gain has been reported after FMT $(57,58)$. Therefore, the question is raised whether transfer of an adult microbiome to a developing microbiome in a child may predispose to quickening of immune aging and development of immune related complications (59). At the current time, these concerns are largely hypothetical and there have been no studies with long term follow up of FMT in children to provide evidence to support or refute this theory. Using age-matched donors, in a similar stage of microbiome development, may address this concern in children and warrants further exploration. Moreover, detailed short-term and longerterm analysis of microbiome changes that occur in children with FMT, along immunological and metabolic consequences, are lacking and are needed to better understand the potentially far reaching impact of FMT in a developing child.

Technical aspects for FMT in children also remained unanswered. The ideal amount of stool to use for children of varying sizes and best mode of delivery needs to be addressed. In addition, potentially different donor screening protocols for infection should be used for FMT in children, for example, for infections which young children are less likely to have been exposed to than adults such as cytomegalovirus (60).

There are currently many studies being conducted examining FMT in children that may help to start to address some of these issues. These include randomized placebo controlled trials of FMT for CDI in children, comparing the use of fresh stool from a related donor to frozen stool from a screened anonymous donor for FMT for CDI in children and also more detailed studies examining the microbiome changes that occur after FMT in children (clinical trials.gov).

\section{CONCLUSIONS}

FMT is an effective treatment for recurrent CDI in adults. There is limited data in children, but initial reports appear encouraging that FMT is effective for recurrent CDI and seems to be safe at least in the short term in IBD. Certainly controlled trials with longer follow up and detailed analysis of the impact on the microbiome of FMT in children would help further address its efficacy and safety. However, it is likely that in this rapidly moving field, FMT may soon be replaced by the oral administration of laboratory-designed bacterial products that achieve similar treatment successes. Nevertheless, in whatever fashion microbiome targeting occurs, manipulation of the intestinal microbiome has the potential to have far reaching implications on both gastrointestinal and nongastrointestinal sites, including the immune system, and the developing microbiome of a child may be particularly vulnerable.

\section{STATEMENT OF FINANCIAL SUPPORT}

No financial assistance was received in support of the study.

Disclosure: None.

\section{REFERENCES}

1. Gill SR, Pop M, Deboy RT, et al. Metagenomic analysis of the human distal gut microbiome. Science 2006;312:1355-9.
2. Qin J, Li R, Raes J, et al.; MetaHIT Consortium. A human gut microbial gene catalogue established by metagenomic sequencing. Nature 2010;464:59-65.

3. Lee YK, Mazmanian SK. Has the microbiota played a critical role in the evolution of the adaptive immune system? Science 2010;330:1768-73.

4. Manichanh C, Borruel N, Casellas F, Guarner F. The gut microbiota in IBD. Nat Rev Gastroenterol Hepatol 2012;9:599-608.

5. Frank DN, St Amand AL, Feldman RA, Boedeker EC, Harpaz N, Pace NR. Molecular-phylogenetic characterization of microbial community imbalances in human inflammatory bowel diseases. Proc Natl Acad Sci USA 2007;104:13780-5.

6. Collins SM. A role for the gut microbiota in IBS. Nat Rev Gastroenterol Hepatol 2014;11:497-505.

7. Tilg H, Kaser A. Gut microbiome, obesity, and metabolic dysfunction. J Clin Invest 2011;121:2126-32.

8. Chang JY, Antonopoulos DA, Kalra A, et al. Decreased diversity of the fecal Microbiome in recurrent Clostridium difficile-associated diarrhea. J Infect Dis 2008;197:435-8.

9. Gough E, Shaikh H, Manges AR. Systematic review of intestinal microbiota transplantation (fecal bacteriotherapy) for recurrent Clostridium difficile infection. Clin Infect Dis 2011;53:994-1002.

10. Lessa FC, Mu Y, Bamberg WM, et al. Burden of Clostridium difficile infection in the United States. N Engl J Med 2015;372:825-34.

11. Ananthakrishnan AN. Clostridium difficile infection: epidemiology, risk factors and management. Nat Rev Gastroenterol Hepatol 2011;8:17-26.

12. van Nood E, Vrieze A, Nieuwdorp M, et al. Duodenal infusion of donor feces for recurrent Clostridium difficile. N Engl J Med 2013;368:407-15.

13. Cammarota G, Masucci L, Ianiro G, et al. Randomised clinical trial: faecal microbiota transplantation by colonoscopy vs. vancomycin for the treatment of recurrent Clostridium difficile infection. Aliment Pharmacol Ther 2015;41:835-43.

14. Sha S, Liang J, Chen M, et al. Systematic review: faecal microbiota transplantation therapy for digestive and nondigestive disorders in adults and children. Aliment Pharmacol Ther 2014;39:1003-32.

15. Houghteling PD, Walker WA. Why is initial bacterial colonization of the intestine important to infants' and children's health? J Pediatr Gastroenterol Nutr 2015;60:294-307.

16. Putignani L, Del Chierico F, Petrucca A, Vernocchi P, Dallapiccola B. The human gut microbiota: a dynamic interplay with the host from birth to senescence settled during childhood. Pediatr Res 2014;76:2-10.

17. Sammons JS, Toltzis P. Recent trends in the epidemiology and treatment of C. difficile infection in children. Curr Opin Pediatr 2013;25:116-21.

18. Wendt JM, Cohen JA, Mu Y, et al. Clostridium difficile infection among children across diverse US geographic locations. Pediatrics 2014;133: 651-8.

19. Nicholson MR, Thomsen IP, Slaughter JC, Creech CB, Edwards KM. Novel risk factors for recurrent Clostridium difficile infection in children. J Pediatr Gastroenterol Nutr 2015;60:18-22.

20. McFarland LV, Elmer GW, Surawicz CM. Breaking the cycle: treatment strategies for 163 cases of recurrent Clostridium difficile disease. Am J Gastroenterol 2002;97:1769-75.

21. Barbut F, Richard A, Hamadi K, Chomette V, Burghoffer B, Petit JC. Epidemiology of recurrences or reinfections of Clostridium difficile-associated diarrhea. J Clin Microbiol 2000;38:2386-8.

22. Hourigan SK, Oliva-Hemker M, Hutfless S. The prevalence of Clostridium difficile infection in pediatric and adult patients with inflammatory bowel disease. Dig Dis Sci 2014;59:2222-7.

23. Kelsen JR, Kim J, Latta D, et al. Recurrence rate of clostridium difficile infection in hospitalized pediatric patients with inflammatory bowel disease. Inflamm Bowel Dis 2011;17:50-5.

24. Walia R, Kunde S, Mahajan L. Fecal microbiota transplantation in the treatment of refractory Clostridium difficile infection in children: an update. Curr Opin Pediatr 2014;26:573-8.

25. Kronman MP, Nielson HJ, Adler AL, et al. Fecal microbiota transplantation via nasogastric tube for recurrent clostridium difficile infection in pediatric patients. J Pediatr Gastroenterol Nutr 2015;60:23-6. 
26. Russell GH, Kaplan JL, Youngster I, et al. Fecal transplant for recurrent Clostridium difficile infection in children with and without inflammatory bowel disease. J Pediatr Gastroenterol Nutr 2014;58:588-92.

27. Pierog A, Mencin A, Reilly NR. Fecal microbiota transplantation in children with recurrent Clostridium difficile infection. Pediatr Infect Dis J 2014;33:1198-200.

28. Walia R, Garg S, Song Y, et al. Efficacy of fecal microbiota transplantation in 2 children with recurrent Clostridium difficile infection and its impact on their growth and gut microbiome. J Pediatr Gastroenterol Nutr 2014;59:565-70.

29. Hourigan SK, Chen LA, Grigoryan Z, et al. Microbiome changes associated with sustained eradication of Clostridium difficile after single faecal microbiota transplantation in children with and without inflammatory bowel disease. Aliment Pharmacol Ther 2015;42:741-52.

30. Kahn SA, Young S, Rubin DT. Colonoscopic fecal microbiota transplant for recurrent Clostridium difficile infection in a child. Am J Gastroenterol 2012;107:1930-1.

31. Wang J, Xiao Y, Lin K, Song F, Ge T, Zhang T. Pediatric severe pseudomembranous enteritis treated with fecal microbiota transplantation in a 13-month-old infant. Biomed Rep 2015;3:173-5.

32. Rubin TA, Gessert CE, Aas J, Bakken JS. Fecal microbiome transplantation for recurrent Clostridium difficile infection: report on a case series. Anaerobe 2013;19:22-6.

33. Kelly CR, Ihunnah C, Fischer M, et al. Fecal microbiota transplant for treatment of Clostridium difficile infection in immunocompromised patients. Am J Gastroenterol 2014;109:1065-71.

34. Jangi S, Lamont JT. Asymptomatic colonization by Clostridium difficile in infants: implications for disease in later life. J Pediatr Gastroenterol Nutr 2010;51:2-7.

35. Schutze GE, Willoughby RE; Committee on Infectious Diseases; American Academy of Pediatrics. Clostridium difficile infection in infants and children. Pediatrics 2013;131:196-200.

36. Hourigan SK, Chirumamilla SR, Ross T, et al. Clostridium difficile carriage and serum antitoxin responses in children with inflammatory bowel disease. Inflamm Bowel Dis 2013;19:2744-52.

37. De Leon LM, Watson JB, Kelly CR. Transient flare of ulcerative colitis after fecal microbiota transplantation for recurrent Clostridium difficile infection. Clin Gastroenterol Hepatol 2013;11:1036-8.

38. Quera R, Espinoza R, Estay C, Rivera D. Bacteremia as an adverse event of fecal microbiota transplantation in a patient with Crohn's disease and recurrent Clostridium difficile infection. J Crohns Colitis 2014;8:252-3.

39. Song Y, Garg S, Girotra M, et al. Microbiota dynamics in patients treated with fecal microbiota transplantation for recurrent Clostridium difficile infection. PLoS One 2013;8:e81330.

40. Shahinas D, Silverman M, Sittler T, et al. Toward an understanding of changes in diversity associated with fecal microbiome transplantation based on 16S rRNA gene deep sequencing. MBio 2012;3:.

41. Grehan MJ, Borody TJ, Leis SM, Campbell J, Mitchell H, Wettstein A. Durable alteration of the colonic microbiota by the administration of donor fecal flora. J Clin Gastroenterol 2010;44:551-61.

42. Seekatz AM, Aas J, Gessert CE, et al. Recovery of the gut microbiome following fecal microbiota transplantation. MBio 2014;5:e0893-14.
43. Colman RJ, Rubin DT. Fecal microbiota transplantation as therapy for inflammatory bowel disease: a systematic review and meta-analysis. J Crohns Colitis 2014;8:1569-81.

44. Moayyedi P, Surette MG, Kim PT, et al. Fecal microbiota transplantation induces remission in patients with active ulcerative colitis in a randomized controlled trial. Gastroenterology 2015;149:102-109.e6.

45. Rossen NG, Fuentes S, van der Spek MJ, et al. Findings from a randomized controlled trial of fecal transplantation for patients with ulcerative colitis. Gastroenterology 2015;149:110-118.e4.

46. Kunde S, Pham A, Bonczyk S, et al. Safety, tolerability, and clinical response after fecal transplantation in children and young adults with ulcerative colitis. J Pediatr Gastroenterol Nutr 2013;56:597-601.

47. Suskind DL, Singh N, Nielson H, Wahbeh G. Fecal microbial transplant via nasogastric tube for active pediatric ulcerative colitis. J Pediatr Gastroenterol Nutr 2015;60:27-9.

48. Suskind DL, Brittnacher MJ, Wahbeh G, et al. Fecal microbial transplant effect on clinical outcomes and fecal microbiome in active Crohn's disease. Inflamm Bowel Dis 2015;21:556-63.

49. Kellermayer R, Nagy-Szakal D, Harris RA, et al. Serial fecal microbiota transplantation alters mucosal gene expression in pediatric ulcerative colitis. Am J Gastroenterol 2015;110:604-6.

50. Vandenplas Y, Veereman G, van der Werff Ten Bosch J et al. Fecal microbial transplantation in a one-year-old girl with early onset colitis - caution advised. J Pediatr Gastroenterol Nutr 2015;61:e12-4.

51. Frank DN, Robertson CE, Hamm CM, et al. Disease phenotype and genotype are associated with shifts in intestinal-associated microbiota in inflammatory bowel diseases. Inflamm Bowel Dis 2011;17:179-84.

52. Bakken JS, Borody T, Brandt LJ, et al.; Fecal Microbiota Transplantation Workgroup. Treating Clostridium difficile infection with fecal microbiota transplantation. Clin Gastroenterol Hepatol 2011;9:1044-9.

53. Sharon I, Morowitz MJ, Thomas BC, Costello EK, Relman DA, Banfield JF. Time series community genomics analysis reveals rapid shifts in bacterial species, strains, and phage during infant gut colonization. Genome Res 2013;23:111-20.

54. Claesson MJ, Jeffery IB, Conde S, et al. Gut microbiota composition correlates with diet and health in the elderly. Nature 2012;488:178-84.

55. Turnbaugh PJ, Ley RE, Mahowald MA, Magrini V, Mardis ER, Gordon JI. An obesity-associated gut microbiome with increased capacity for energy harvest. Nature 2006;444:1027-31.

56. Cox LM, Yamanishi S, Sohn J, et al. Altering the intestinal microbiota during a critical developmental window has lasting metabolic consequences. Cell 2014;158:705-21.

57. Brandt LJ, Aroniadis OC, Mellow M, et al. Long-term follow-up of colonoscopic fecal microbiota transplant for recurrent Clostridium difficile infection. Am J Gastroenterol 2012;107:1079-87.

58. Alang N, Kelly CR. Weight gain after fecal microbiota transplantation. Open Forum Infect Dis 2015;2:ofv004.

59. Lynch SV. Fecal Microbiota Transplantation for Recurrent Clostridium difficile Infection in Pediatric Patients: Encouragement Wrapped in Caution. J Pediatr Gastroenterol Nutr 2015;60:1-3.

60. Staras SA, Dollard SC, Radford KW, Flanders WD, Pass RF, Cannon MJ. Seroprevalence of cytomegalovirus infection in the United States, 19881994. Clin Infect Dis 2006;43:1143-51. 